\title{
Orbit, emission spectrum, and photometric analysis of two flickering sporadic fireballs
}

\author{
J. M. Madiedo ${ }^{1,2}$, J. M. Trigo-Rodríguez ${ }^{3}$, N. Konovalova ${ }^{4}$, J. L. Ortiz ${ }^{5}$, A. J. Castro-Tirado ${ }^{5}$, J. Alonso-Azcárate ${ }^{6}$ \\ J. Lacruz ${ }^{7}$, and J. Cabrera-Caño ${ }^{2}$ \\ 1 Facultad de Ciencias Experimentales, Universidad de Huelva, 21071 Huelva, Spain \\ e-mail: jmmadiedo@gmail.com \\ 2 Departamento de Física Atómica, Molecular y Nuclear, Facultad de Física, Universidad de Sevilla, 41012 Sevilla, Spain \\ 3 Institut de Ciències de l'Espai (CSIC-IEEC), Campus UAB, Facultat de Ciències, Torre C5-parell-2 ${ }^{\mathrm{a}}$, 08193 Bellaterra, Barcelona, \\ Spain \\ 4 Institute of Astrophysics of the Academy of Sciences of the Republic of Tajikistan, Bukhoro, str. 22, 734042 Dushanbe, Tajikistan \\ 5 Instituto de Astrofísica de Andalucá, CSIC, Apt. 3004, 18080 Granada, Spain \\ ${ }^{6}$ Universidad de Castilla-La Mancha, 45071 Toledo, Spain \\ 7 La Cañada Observatory (MPC J87), Ávila, Spain
}

Received 22 April 2013 / Accepted 4 June 2013

\section{ABSTRACT}

\begin{abstract}
We present the observations of two fireballs that exhibit fast and quasi-periodic brightness variations along their atmospheric path. This phenomenon, which is known as "flickering", is associated to the entry of spinning non-spherical meteoroids into the atmosphere. Both bolides were imaged in the framework of the continuous fireball monitoring and meteor spectroscopy campaigns organized by the SPanish Meteor Network (SPMN) in 2012. Their atmospheric trajectory, radiant, and orbit were calculated. The preatmospheric mass and tensile strength of the parent meteoroids were also estimated, and the rotation of these bodies analyzed on the basis of the photometric behavior of the bolides. Although small (less than 80 grams), a non-zero terminal mass was determined for both events. The emission spectra produced during the ablation of the meteoroids in the atmosphere are also discussed.
\end{abstract}

Key words. meteorites, meteors, meteoroids

\section{Introduction}

Classical meteor light curves have the basic shape of a parabola that is skewed about the point of maximum brightness (Hughes 1978). Most bright meteor light curves approximately fit this description, with events produced by compact meteoroids exhibiting their maximum luminosity during the second half of their trajectory (Murray et al. 1999; Campbell et al. 2000) and dustball meteoroids showing this maximum earlier (Hawkes \& Jones 1975). However, about $5 \%$ of observed fireball events exhibit a distinct brightness modulation called flickering (Opik 1936; Beech \& Brown 2000), so that they show fast quasi-periodic, small-amplitude brightness variations superimposed upon a classical light curve. This phenomenon is indicative of the parent meteoroid being both non-spherical and rotating and, so, the analysis of the light curve of flickering fireballs can provide information about the spin and shape of these particles (Beech 2001).

Here we present the results of the analysis of two flickering bolides recorded over the Iberian Peninsula in 2012 in the framework of our continuous fireball monitoring and meteor spectroscopy campaigns. These bright sporadic events, with absolute magnitudes of $-16.5 \pm 0.5$ and $-10.0 \pm 0.5$ were imaged on July 21 and December 29, respectively. For identification in our database they were assigned a SPanish Meteor Network (SPMN) code established after the recording date with format yymmdd. Besides this, the bolides were named according to the geographical location nearest to the projection on the ground of their atmospheric trajectory. Thus, bolide SPMN210712 was named "Fuencaliente" after this village located in Andalusia. On the other hand, the fireball SPMN2191212 was named "Sevilla", because the meteoroid disintegrated over this city in the south of Spain.

\section{Instrumentation and data reduction methods}

The geographical coordinates of the meteor-observing stations that imaged the bolides analyzed here are shown in Table 1. From these we operate high-sensitivity CCD video devices (models $902 \mathrm{H}$ and $902 \mathrm{H}$ Ultimate from Watec Corporation, Japan) to monitor the night sky. These generate interlaced imagery according to the PAL video standard. Thus, they produce video files at a rate of 25 frames per second and with a resolution of $720 \times 576$ pixels. The operation of these video stations is given in Madiedo \& Trigo-Rodríguez (2008, 2010), Trigo-Rodriguez et al. (2007, 2009), and Madiedo et al. (2010). For meteor spectroscopy, holographic diffraction gratings (500 or 1000 lines $/ \mathrm{mm}$, depending on the device) are attached to the objective lens of some of these CCD cameras.

For data reduction, we first deinterlaced the video sequences provided by our CCD cameras. Thus, even and odd fields were separated for each video frame, and a new video file containing these was generated. Since this operation implies duplicating the total number of frames in the original sequence, the frame rate in the resulting video was transformed from 25 to $50 \mathrm{fps}$. Then, an astrometric measurement was done by hand in order to obtain 
Table 1. Geographical coordinates of the meteor observing stations involved in this work.

\begin{tabular}{lcccc}
\hline \hline Station \# & Station name & Latitude (N) & Longitude $(\mathrm{W})$ & Alt. (m) \\
\hline 1 & Sevilla & $05^{\circ} 58^{\prime} 50^{\prime \prime}$ & $37^{\circ} 20^{\prime} 46^{\prime \prime}$ & 28 \\
2 & La Hita & $03^{\circ} 11^{\prime} 00^{\prime \prime}$ & $39^{\circ} 34^{\prime} 06^{\prime \prime}$ & 674 \\
3 & Huelva & $06^{\circ} 56^{\prime} 11^{\prime \prime}$ & $37^{\circ} 15^{\prime} 10^{\prime \prime}$ & 25 \\
4 & Sierra Nevada & $03^{\circ} 23^{\prime} 05^{\prime \prime}$ & $37^{\circ} 03^{\prime} 51^{\prime \prime}$ & 2896 \\
5 & El Arenosillo & $06^{\circ} 43^{\prime} 58^{\prime \prime}$ & $37^{\circ} 06^{\prime} 16^{\prime \prime}$ & 40 \\
6 & Toledo & $03^{\circ} 57^{\prime} 29^{\prime \prime}$ & $39^{\circ} 49^{\prime} 30^{\prime \prime}$ & 639 \\
7 & Ávila & $04^{\circ} 29^{\prime} 30^{\prime \prime}$ & $40^{\circ} 36^{\prime} 18^{\prime \prime}$ & 1400 \\
\hline
\end{tabular}

Table 2. Brightness, trajectory and radiant data (J2000) for the fireballs analyzed in this work.

\begin{tabular}{lccccccccccc}
\hline \hline $\begin{array}{l}\text { SPMN code } \\
\text { and name }\end{array}$ & Date & $\begin{array}{c}\text { Time (UTC) } \\
\pm 0.1 \mathrm{~s}\end{array}$ & $M$ & $\begin{array}{c}H_{\mathrm{b}} \\
(\mathrm{km})\end{array}$ & $\begin{array}{c}H_{\mathrm{m}} \\
(\mathrm{km})\end{array}$ & $\begin{array}{c}H_{\mathrm{e}} \\
(\mathrm{km})\end{array}$ & $\begin{array}{c}\alpha_{\mathrm{g}} \\
\left({ }^{\circ}\right)\end{array}$ & $\begin{array}{c}\delta_{\mathrm{g}} \\
\left({ }^{\circ}\right)\end{array}$ & $\begin{array}{c}V_{\text {inf }} \\
\left(\mathrm{km} \mathrm{s}^{-1}\right)\end{array}$ & $\begin{array}{c}V_{\mathrm{g}} \\
\left(\mathrm{km} \mathrm{s}^{-1}\right)\end{array}$ & $\begin{array}{c}V_{\mathrm{h}} \\
\left(\mathrm{km} \mathrm{s}^{-1}\right)\end{array}$ \\
\hline 210712 & Jul. 21, & $22 \mathrm{~h} 21 \mathrm{~m} 56.7 \mathrm{~s}$ & -16.5 & 81.8 & 43.5 & 29.0 & 291.6 & 39.0 & 19.4 & 15.8 & 32.6 \\
"Fuencaliente" & 2012 & & \pm 0.5 & \pm 0.5 & \pm 0.5 & \pm 0.5 & \pm 0.5 & \pm 0.4 & \pm 0.3 & \pm 0.3 & \pm 0.3 \\
291212 & Dec. 29, & $23 \mathrm{~h} 42 \mathrm{~m} 07.6 \mathrm{~s}$ & -10.0 & 89.6 & 44.5 & 30.7 & 44.3 & 45.7 & 16.9 & 13.0 & 39.5 \\
"Sevilla" & 2012 & & \pm 0.5 & \pm 0.5 & \pm 0.5 & \pm 0.5 & \pm 0.3 & \pm 0.3 & \pm 0.3 & \pm 0.3 & \pm 0.3 \\
\hline
\end{tabular}

Notes. The meaning of the symbols employed here is provided in Sect. 3.

Table 3. Orbital elements (J2000) and Tisserand parameter with respect to Jupiter for the bolides discussed in the text.

\begin{tabular}{|c|c|c|c|c|c|c|c|}
\hline $\begin{array}{l}\text { SPMN code } \\
\text { and name }\end{array}$ & $\begin{array}{c}a \\
(\mathrm{AU})\end{array}$ & $e$ & $\begin{array}{c}i \\
\left(^{\circ}\right) \\
\end{array}$ & $\begin{array}{l}\Omega \\
\left({ }^{\circ}\right)\end{array}$ & $\begin{array}{l}\omega \\
\left(^{\circ}\right) \\
\end{array}$ & $\begin{array}{c}q \\
(\mathrm{AU})\end{array}$ & $T_{\mathrm{J}}$ \\
\hline 210712 & 1.30 & 0.32 & 25.6 & 119.3604 & 242.8 & 0.879 & 4.83 \\
\hline "Fuencaliente" & \pm 0.02 & \pm 0.01 & \pm 0.5 & $\pm 10^{-4}$ & \pm 1.7 & \pm 0.004 & \pm 0.06 \\
\hline 291212 & 3.7 & 0.75 & 9.0 & 278.5108 & 209.1 & 0.929 & 2.50 \\
\hline "Sevilla" & \pm 0.3 & \pm 0.02 & \pm 0.2 & $\pm 10^{-4}$ & \pm 0.3 & \pm 0.002 & \pm 0.08 \\
\hline
\end{tabular}

the plate $(x, y)$ coordinates of the meteor along its apparent path from each station. The astrometric measurements were then introduced in our Amalthea software (Trigo-Rodríguez et al. 2009; Madiedo et al. 2011a), which transforms plate coordinates into equatorial coordinates by using the position of reference stars appearing in the images. This package employs the method of intersecting planes to determine the position of the apparent radiant and also to reconstruct the trajectory in the atmosphere of meteors recorded from at least two different observing stations (Ceplecha 1987). In this way, the beginning and terminal heights of the meteor are inferred. From the sequential measurements of the video frames and the trajectory length, the velocity of the meteor along its path is obtained. The preatmospheric velocity $V_{\infty}$ is found by measuring the velocities at the earliest part of the meteor trajectory. Once these data are known, the software computes the orbital parameters of the corresponding meteoroid by following the procedure described in Ceplecha (1987).

\section{Observations: atmospheric trajectory and orbit}

From calculation of the atmospheric trajectory of both fireballs, we obtained the beginning $\left(H_{\mathrm{b}}\right)$, ending $\left(H_{\mathrm{e}}\right)$, and maximum brightness $\left(H_{\mathrm{m}}\right)$ heights for each event. Their absolute magnitude $(M)$ was obtained by comparing the brightness level of the pixels near the maximum luminosity of the meteor trail and those of stars appearing within the field of view. These characteristics of the fireball path are listed in Table 2. The planes intersection method also provided the location of the apparent radiant for each fireball and the preatmospheric velocity $V_{\infty}$ of the parent meteoroids. Once this information was known, we calculated the orbital elements in the solar system of these particles (Table 3).
The characteristics and specific circumstances of both events are detailed below.

\subsection{The "Fuencaliente" sporadic event (SPMN210712)}

This deep-penetrating fireball was observed on July 21, 2012 at $22 \mathrm{~h} 21 \mathrm{~m} 56.7 \mathrm{~s} \pm 0.1 \mathrm{~s}$ UTC (about $22 \mathrm{~min}$ after local midnight). It lasted over three seconds and was widely witnessed over Spain. Thus, because of the very high brightness of this bolide (its estimated absolute magnitude is $-16.5 \pm 0.5$ ) and excellent weather conditions on the Iberian Peninsula, the fireball was imaged from most SPMN stations located in the south and center of Spain (Fig. 1). The best images, however, were obtained from our fireball monitoring systems operating from $\mathrm{La}$ Hita Astronomical Observatory, Toledo, El Arenosillo, Sevilla, Ávila, and Huelva. The emission spectrum produced during the ablation of the object that produced this event was also recorded by two video spectrographs operating from El Arenosillo and La Hita. Our calculations reveal that the meteoroid struck the atmosphere with an initial velocity $V_{\infty}=19.4 \pm 0.3 \mathrm{~km} \mathrm{~s}^{-1}$. The luminous phase began at a height of $81.8 \pm 0.5 \mathrm{~km}$ and ended at $29.0 \pm 0.5 \mathrm{~km}$ above the ground level. The angle of the atmospheric trajectory to the Earth's surface was of about $74.3^{\circ}$ and the apparent radiant was located at the position $\alpha=292.0 \pm 0.5^{\circ}$, $\beta=38.9 \pm 0.4^{\circ}$. With this information, the heliocentric orbit of the parent meteoroid was obtained. The projection of this orbit on the ecliptic plane is plotted in Fig. 1c. The calculated orbital parameters (Table 3) confirmed the sporadic nature of the event, but also the likely asteroidal origin of the meteoroid (Tisserand parameter with respect to Jupiter $T_{\mathrm{J}}=4.83 \pm 0.06$ ). 


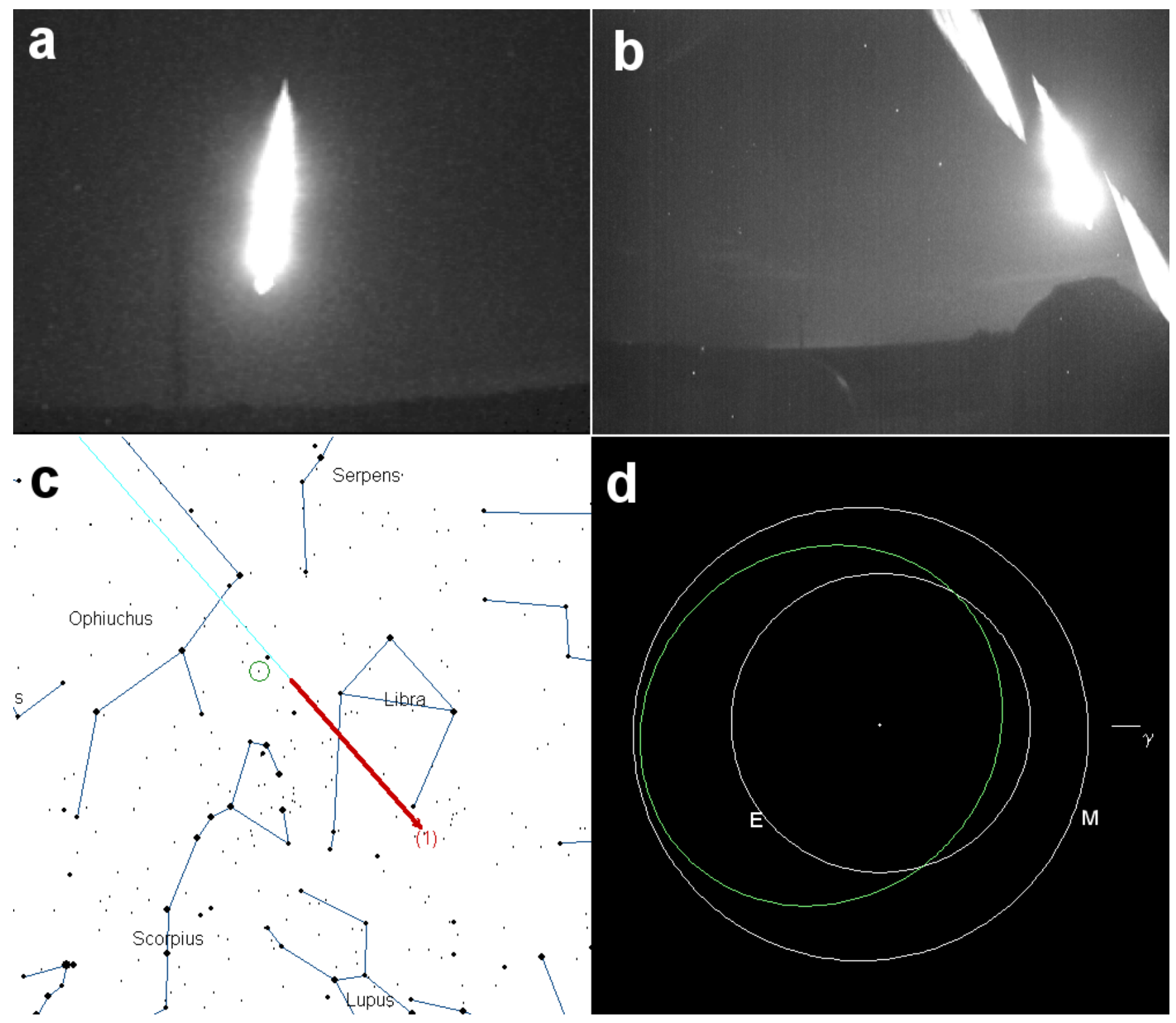

Fig. 1. a) Composite image of the "Fuencaliente" fireball (code SPMN210712), imaged on July 21, 2012 at $22 \mathrm{~h} 21 \mathrm{~m} 56.7 \pm 0.1 \mathrm{~s}$ UTC from a) El Arenosillo and b) La Hita Astronomical Observatory. c) Apparent trajectory of the bolide as seen from La Hita. d) Heliocentric orbit of the meteoroid projected on the ecliptic plane.

\subsection{The "Sevilla" fireball (SPMN291212)}

A slow-moving fireball was imaged on December 29, 2012, at 23h42m07.6 $\pm 0.1 \mathrm{~s}$ UTC from stations \#1 and \#5 (Fig. 2). The event lasted about $4.7 \mathrm{~s}$, and the photometric analysis of the video sequences recorded by our CCD cameras indicates that it reached an absolute magnitude of $-10.0 \pm 0.5$ at its brightest phase. Its apparent trajectory in the sky as seen from both meteor observing stations is shown in Fig. 2c. The bolide began at $89.6 \pm 0.5 \mathrm{~km}$ above the ground level, with the terminal point located at $30.7 \pm 0.5 \mathrm{~km}$. The angle of the atmospheric path to the Earth's surface was of about $62.8^{\circ}$. The position of the apparent radiant was $\alpha=44.3 \pm 0.3^{\circ}, \beta=45.7 \pm 0.3^{\circ}$. The analysis of the atmospheric trajectory also reveals that the parent meteoroid impacted the atmosphere with an initial velocity $V_{\infty}=16.9 \pm 0.3 \mathrm{~km} \mathrm{~s}^{-1}$. From the calculation of the orbital elements of this particle (Table 3) we infer that the meteoroid was following a Jupiter Family Comet orbit before impacting the atmosphere $\left(T_{\mathrm{J}}=2.50 \pm 0.08\right)$. This orbit is shown in Fig. $2 \mathrm{~d}$.

\section{Results and discussion}

\subsection{Photometric analysis}

The photometric analysis of the video sequences recorded for each fireball provided the light curves shown in Figs. 3 and 4. As can be noticed, both events exhibit their maximum brightness during the second half of their trajectory, a behavior that is typical of fireballs produced by compact meteoroids (Murray et al. 1999; Campbell et al. 2000). The flickering effect is also obvious, as both light curves reveal rapid and quasi-periodic oscillations in luminosity.

One of the parameters that can be obtained from these light curves is the preatmospheric mass of the meteoroids. Thus, this photometric mass mp can be obtained as the total mass lost due to the ablation process between the beginning of the luminous phase and the terminal point of the atmospheric trajectory,

$m_{\mathrm{p}}=2 \int_{t_{\mathrm{b}}}^{t_{\mathrm{e}}} \frac{I}{\tau v^{2}} \mathrm{~d} t$ 


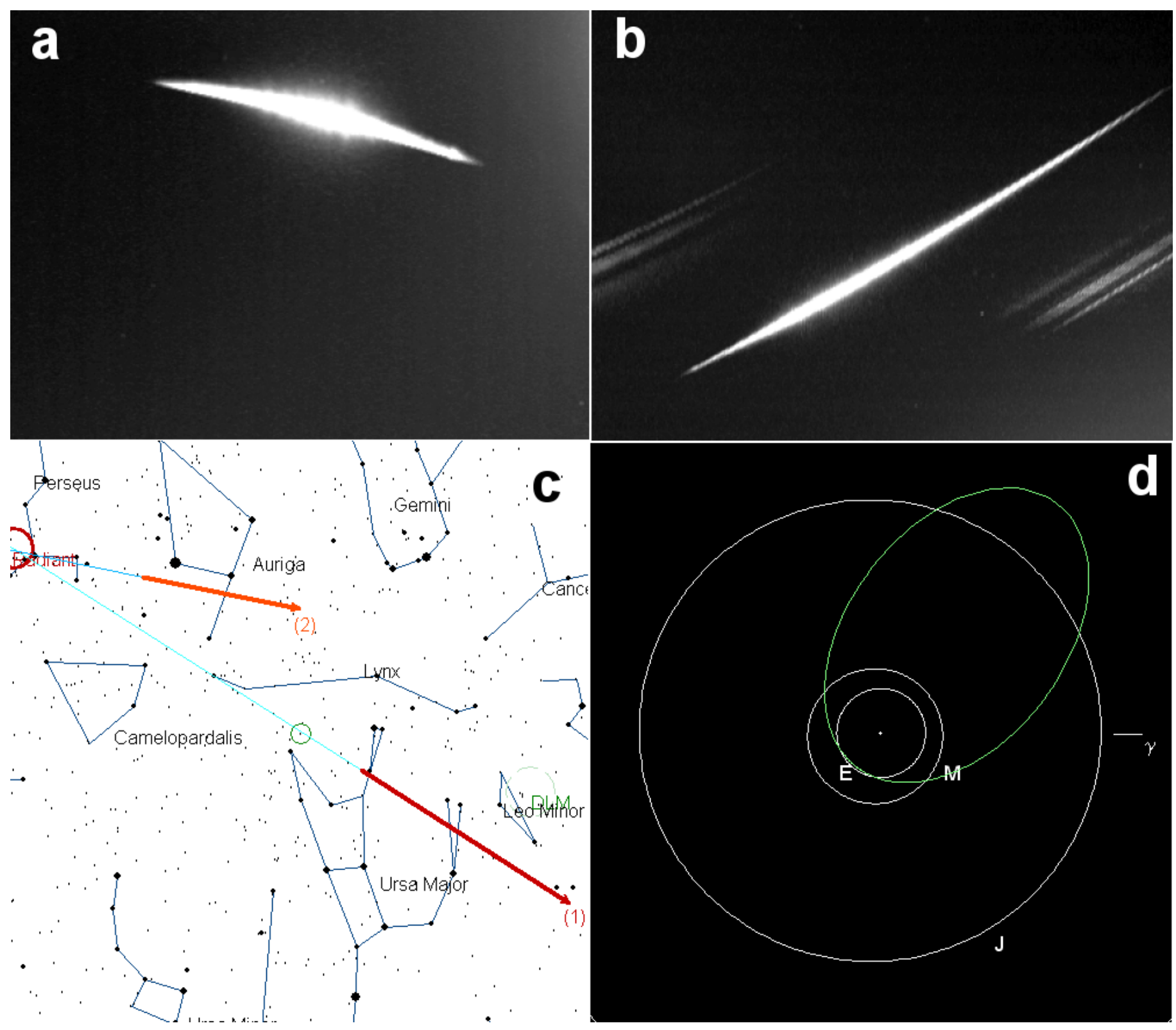

Fig. 2. Composite image of the "Sevilla" sporadic fireball (code SPMN291212), imaged on Dec. 29, 2012 at 23h42m07.6 $\pm 0.1 \mathrm{~s}$ UTC from a) Sevilla and b) El Arenosillo. c) Apparent trajectory of the bolide as seen (1) El Arenosillo and (2) Sevilla. d) Heliocentric orbit of the meteoroid projected on the ecliptic plane.

where $t_{\mathrm{b}}$ and $t_{\mathrm{e}}$ are, respectively, the times corresponding to the beginning and the end of the luminous phase, and $\mathrm{I}$ is the measured luminosity of the fireball, which is related to the absolute magnitude $\mathrm{M}$ of the event by means of the equation

$I=10^{-0.4 M}$.

The velocity-dependent luminous efficiency $(\tau)$ was calculated according to the relationships given by Ceplecha \& McCrosky (1976). In this way, the calculation of the initial mass yields $212 \pm 20 \mathrm{~kg}$ for the "Fuencaliente" meteoroid and $4.1 \pm$ $0.5 \mathrm{~kg}$ for the body producing the "Sevilla" event. The diameter of these particles would be $55 \pm 3$ and $14.8 \pm 1.5 \mathrm{~cm}$, respectively, for a bulk density $\rho_{\mathrm{m}}=2.4 \mathrm{~g} \mathrm{~cm}^{-3}$. For a density $\rho_{\mathrm{m}}=3.7 \mathrm{~g} \mathrm{~cm}^{-3}$, the calculated diameter yields $47 \pm 2 \mathrm{~cm}$ for the "Fuencaliente" meteoroid and $12.8 \pm 1.5 \mathrm{~cm}$ for "Sevilla".

Observational data show that both, sporadic bright fireballs (Getman 1993; Brown et al. 1998; Beech 2001) but also bright bolides belonging to annual meteor showers such as the Geminids (Halliday 1963; Babadzhanov \& Konovalova 2004) and the Taurids (Konovalova 2003) show quasi-periodic brightness variations during the flight in the Earth's atmosphere. The flickering frequencies reported in the literature vary from a few $\mathrm{Hz}$ (low-frequency flickering) to about $500 \mathrm{~Hz}$ (high-frequency flickering). The flickering phenomenon is apparently associated with meteoroid ablation and can provide information about the spin and shape of the parent meteoroid, but also about its physical properties, mainly the density, strength, and structure. Some authors argued that this effect is a manifestation of the rotational modulation of surface mass loss during the ablation of non-spherical meteoroids (Kruchinenko 1995; Beech 2001). Undoubtedly, meteoroids can gain rotation before penetrating the Earth's atmosphere by means of, for instance, collisions taking place in space. According to Olsson-Steel (1987), the angular frequency of these rotations decreases in inverse proportion 


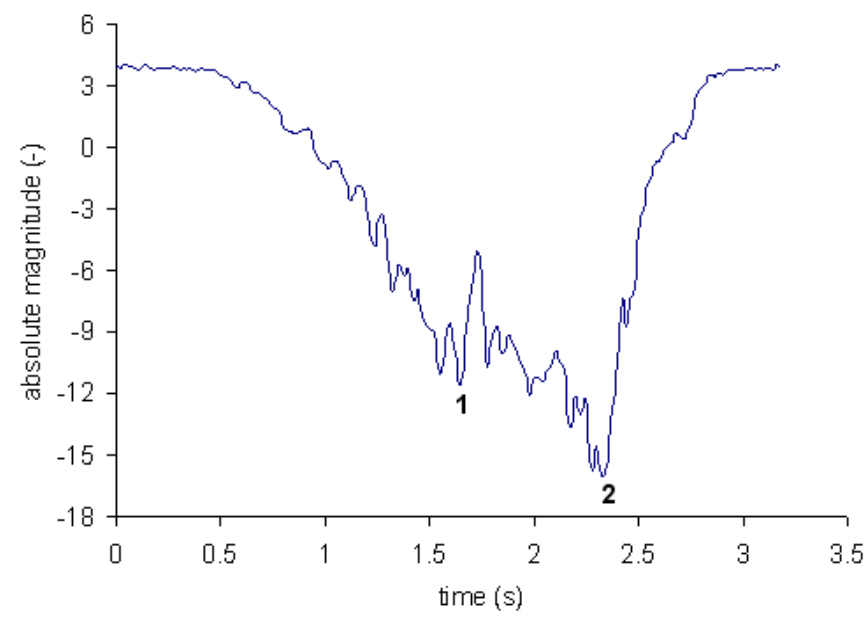

Fig. 3. Light curve of the SPMN210712 "Fuencaliente" fireball.

to the second power of the radius of these bodies. Adolfsson (1994) proposed a method for calculating the initial rotation of large asymmetrical bodies. It must also be mentioned that, in the long term, the so-called Yarkovsky-O'Keefe-RadzievskiiPaddack (YORP) effect can also significantly change the rotational state of meteoroids and small asteroids (Čapek \& Vokrouhlický 2004). On the other hand, other authors proposed that the flickering phenomenon can occur when the pressure of saturated vapors of meteor material and the outer pressure regulate each other automatically (Babadzhanov \& Konovalova 2004). This is, in fact, one of the main possible mechanisms for meteor flickering listed in (Oleak 1964) and presented as responsible for the autofluctuating character of the evaporation process.

For the "Sevilla" fireball, the observed flickering modulation amplitude is $\Delta M \approx 1$ and appears to be almost constant along the atmospheric trajectory of the bolide (Fig. 4). We have analyzed the flickering behavior exhibited by this event on the basis of the quasi-continuous fragmentation (QCF) theory (Novikov et al. 1998). According this model, the equation that provides the intensity $I(t)$ of the light emitted by the fireball can be written as

$$
I(t)=\frac{\tau}{2} v^{2}(t) \Theta\left(T_{\mathrm{e}}-t\right) \int_{\left(t-\tau_{\mathrm{b}}\right) \theta\left(t-\tau_{\mathrm{b}}\right)}^{t} N\left(t_{0}^{\prime}\right) \mathrm{d} m_{\mathrm{f}} / \mathrm{d} t \Theta\left(\tau_{0}-t_{0}^{\prime}\right) \mathrm{d} t_{0}^{\prime}
$$

with

$$
\begin{aligned}
N\left(t_{0}^{\prime}\right) & =\frac{1}{m_{\mathrm{f}}}\left(\mathrm{d} M / \mathrm{d} t_{0}^{\prime}\right) \\
\mathrm{d} M / \mathrm{d} t_{0}^{\prime} & =\frac{\Lambda S}{2} Q_{\mathrm{f}}\left(M / \rho_{\mathrm{m}}\right)^{2 / 3} \rho_{\mathrm{a}} v^{3} \\
\mathrm{~d} m_{\mathrm{f}} / \mathrm{d} t & =\frac{\Lambda^{\prime} S^{\prime}}{2}\left(Q-Q_{\mathrm{f}}\right)\left(m_{\mathrm{f}} / \rho_{\mathrm{f}}\right)^{2 / 3} \rho_{\mathrm{a}} v^{3}
\end{aligned}
$$

where $v$ is the velocity of meteoroid, $M$ and $m_{\mathrm{f}}$ are the meteoroid mass and the initial masses of the smaller fragments at the instant $t_{0}^{\prime}$, respectively.

In Eqs. (3)-(6) the velocity-dependent luminous efficiency $\tau$ is given by Ceplecha \& McCrosky (1976), and $\Lambda, S$, and $\rho_{\mathrm{m}}$ are the heat transfer coefficient, the shape factor, and the bulk density of meteoroid, respectively. On the other hand, $\Lambda^{\prime}, S^{\prime}$, and $\rho_{\mathrm{f}}$ represent these same physical quantities for fragments, and $Q$ and $Q_{\mathrm{f}}$ are the energies of evaporation and fragmentation, respectively, with $\rho_{\mathrm{a}}$ the atmospheric density. The parameter $T_{\mathrm{e}}$ is

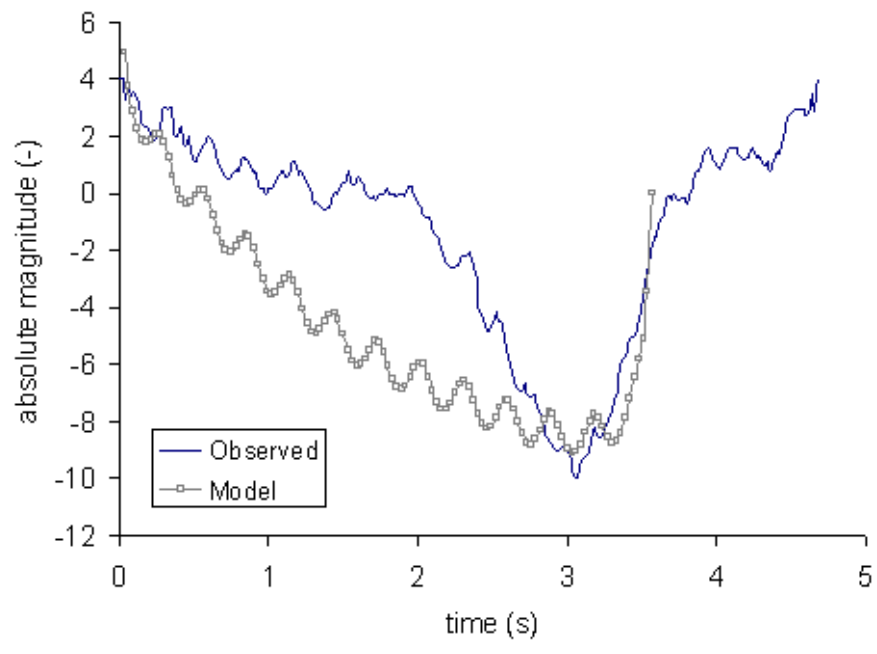

Fig. 4. Light curve of the SPMN291212 "Sevilla" fireball. The modeled light curve according to the QCF model is also included.

defined as $T_{\mathrm{e}}=\tau_{0}+\tau_{\mathrm{e}}$, where $\tau_{0}$ is the lifetime of the meteoroid to complete fragmentation, and $\tau_{\mathrm{e}}$ is the lifetime of a fragment that was detached at the end of fragmentation until its full evaporation. On the other hand, $\tau_{\mathrm{b}}$ is the lifetime of the fragment released at the instant of beginning of fragmentation, and $\tau_{\mathrm{f}}$ is the lifetime of the individual fragment counted from $t_{0}^{\prime}$ until its full evaporation.

Equations (3) to (6) have been solved numerically. We have considered the meteoroid as an ellipsoid of rotation with semimajor axis $a$, semiminor axis $b$ and eccentricity $e^{2}=1-(b / a)^{2}$, which is spinning about its minor axis during its flight in the atmosphere and, consequently, has a time-varying cross-sectional area $A(t)$. We also assumed spherical symmetry for small fragments, so that their shape factor is $S^{\prime}=1.21$. For the heat transfer coefficient of these fragments, we employed $\Lambda^{\prime}=1$, while the heat transfer coefficient was $\Lambda=0.05$ for the meteoroid. In fact, this coefficient depends on the flow regime, as it is conditioned by the thermal shielding of flying away (jumping aside) air molecules and ablated material from the meteoroid. For large meteoroids (with diameters $>10 \mathrm{~cm}$ ) this shielding is strong and then $\Lambda=0.05$ or even lower. For small particles, however, the shielding effect can be neglected and then $\Lambda=1$ (Bronshten 1981). In addition, in our numerical model we have adopted the bulk density-dependent energy of fragmentation $Q_{\mathrm{f}}$ given by Lebedinets (1986). Besides, the shape factor $S$ of the meteoroid was expressed in terms of its volume and time-varying crosssection A(t) given by (Beech 2001):

$A(t)=\pi b^{2}\left[\cos ^{2} \omega+(a / b) \sin ^{2} \omega\right]$

where $\omega=(2 \pi / P) t+\Phi$, with P being the rotation period of the meteoroid, $\Phi$ the rotation phase term, and $t$ the time since the beginning of the simulation.

Figure 4 shows the observed and modeled light curves produced for a spinning meteoroid. The assumed axial ratio corresponds to $b / a=1 / 1.5$, with a rotation period $P=0.58 \mathrm{~s}$, phase $\Phi=0$, and bulk density of meteoroid material $\rho_{\mathrm{m}}=$ $3.2 \pm 0.2 \mathrm{~g} \mathrm{~cm}^{-3}$. As can be noticed, both curves exhibit an almost synchronous amplitude brightness modulation. This correlation between the observed and modeled light curves is the evidence that the flickering effect exhibited by the "Sevilla" event is actually related to the instantaneous rotation rate of the parent meteoroid. 
Table 4. Aerodynamic pressure for flares and break-up processes discussed in the text.

\begin{tabular}{lcccc}
\hline \hline SPMN code and name & Flare \# & Height $(\mathrm{km})$ & Velocity $\left(\mathrm{km} \mathrm{s}^{-1}\right)$ & Aerodynamic pressure $\left(\right.$ dyn $\left.\mathrm{cm}^{-2}\right)$ \\
\hline SPMN210712 "Fuencaliente" & 1 & $55.8 \pm 0.5$ & $15.9 \pm 0.3$ & $(1.2 \pm 0.4) \times 10^{6}$ \\
SPMN291212 "Sevilla" & 2 & $43.5 \pm 0.5$ & $15.3 \pm 0.3$ & $(5.4 \pm 0.4) \times 10^{6}$ \\
\hline
\end{tabular}

On the other hand, the light curve of the "Fuencaliente" fireball (Fig. 3) could not be modeled by means of the QCF or single-body theory. Nevertheless, this light curve reveals a flickering frequency of about $10 \mathrm{~Hz}$, with a maximum flickering modulation amplitude $\Delta M$ of around $2.0 \mathrm{mag}$. By assuming a homogeneous ellipsoidal profile for the meteoroid, the flickering amplitude would vary according to (Beech et al. 2003)

$\Delta M=2.5 \log (a / b)$

with $a$ and $b$ being, respectively, the semimajor and semiminor axes of the meteoroid. Thus, an axis ratio $a / b=6.3$ is required to account for the observed maximum amplitude, so, according to this, the meteoroid would be significantly elongated. However as can be seen in Fig. 3, no significant flickering is exhibited in the beginning of the atmospheric path, which suggests that the rotational state of the meteoroid before impacting the atmosphere is not the main reason for the observed flickering behavior, and the calculated a/b ratio would be unrealistic.

On the other hand, the observed flickering amplitude at times $t>0.7$ seconds cannot be explained on the basis of an increase in the rotation rate in the atmosphere, since this mechanism is not effective for large meteoroids. Thus, for instance, Geminid meteoroids gain rotation in the atmosphere when their initial mass is below $6 \times 10^{-7} \mathrm{~g}$ (Babadzhanov \& Konovalova 2004). On the other hand, Fig. 3 also shows that the flickering effect practically disappears after the main fulguration. In this case, the most likely explanation for the flickering behavior observed for the "Fuencaliente" fireball would be a mechanism of successive detachment of fragments (wreckages) (Oleak 1964; Konovalova 2003).

\subsection{Tensile strength}

The compactness inferred for the meteoroids on the basis of the above-discussed photometric behavior can be confirmed by the estimation of the tensile strength of these particles. Thus, as can be noticed in the composite images shown in Figs. 1 and 2 and also in the light curves plotted in Figs. 3 and 4, the fireballs exhibited at least one very bright flare along their atmospheric path. These events are typically produced by the fragmentation of the meteoroids when these particles penetrate denser atmospheric regions. Thus, once the overloading pressure becomes higher than the particle strength, the particle breaks apart. Quickly after that, a bright flare is produced as a consequence of the fast ablation of tiny fragments delivered to the thermal wave in the fireball's bow shock. The so-called tensile (aerodynamic) strength $S$ at which these breakups take place can be calculated by means of the following equation (Bronshted 1981):

$S=\rho_{\mathrm{a}} v^{2}$

where $v$ is the velocity of the meteoroid at the disruption point and $\rho_{\mathrm{a}}$ the atmospheric density at the height where the fracture takes place. The density can be calculated, for instance, by employing the US standard atmosphere model (US Standard Atmosphere 1976). This aerodynamic strength $S$ can be used as an estimation of the tensile strength of the meteoroid (Trigo-Rodríguez \& Llorca 2006, 2007). The values obtained for the fireballs analyzed in this work are summarized in Table 4, together with the corresponding heights and velocities.

As Fig. 3 shows, the SPMN210712 "Fuencaliente" fireball experienced a big flare at the instant $t_{1}=1.7 \mathrm{~s}$ after the event started its luminous phase, followed by a second and more intense flare at the instant $t_{2}=2.3 \mathrm{~s}$. These events are indicated in Fig. 3 with labels (1) and (2), respectively. The images reveal that the corresponding fragmentations were not catastrophic, as the remaining material continued penetrating the atmosphere. These break-ups took place, respectively, at a height of $55.8 \pm 0.5$ and $43.5 \pm 0.5 \mathrm{~km}$ above the ground level. In this way, we infer that the meteoroid exhibited the first flare under a dynamic pressure of $(1.2 \pm 0.4) \times 10^{6} \mathrm{dyn}^{-2}$, although the main flare took place at $(5.4 \pm 0.4) \times 10^{6} \mathrm{dyn}^{-2}$. These values are higher than the typical strengths found for meteoroids from cometary streams (between $\sim 4.0 \times 10^{3}$ and $\sim 3.4 \times 10^{5} \mathrm{dyn}^{-2}$ according to Trigo-Rodríguez \& Llorca 2006, 2007), which is consistent with the likely asteroidal nature of the meteoroid inferred from the analysis of its orbital parameters.

The brightest phase of the "Sevilla" SPMN291212 fireball took place at a height of $44.5 \pm 0.5 \mathrm{~km}$ above the ground level, when its velocity was $12.9 \pm 0.3 \mathrm{~km} \mathrm{~s}^{-1}$. In this way, according to Eq. (9), the aerodynamic pressure at this point yields $(3.3 \pm 0.4) \times 10^{5} \mathrm{dyn}^{-2}$. When this value is compared with the tensile strength found for meteoroids from cometary streams (Trigo-Rodríguez \& Llorca 2006, 2007), we conclude that the "Sevilla" meteoroid is also tough, exhibiting a similar behavior to meteoroids from evolved cometary bodies such as the Taurids $\left(S \sim 3.4 \times 10^{5} \mathrm{dyn}^{-2}\right.$ ), whose parent body is the Jupiter family comet 2P/Encke (Jenniskens 2006).

\subsection{Terminal mass and meteorite survival}

By taking the calculated values of the ending height of their luminous trajectory into account, the possibility that these deeppenetrating fireballs could produce meteorites was considered. For this purpose the mass of the progenitor body at the terminal point of the luminous atmospheric path was calculated. Thus, the meteoroid mass surviving the ablation process was obtained from the following equation (Ceplecha et al. 1983):

$$
m_{\mathrm{E}}=\frac{1.2 \rho_{\mathrm{E}} v_{\mathrm{E}}^{2}}{(\mathrm{~d} v / \mathrm{d} t)_{\mathrm{E}} \rho_{\mathrm{m}}^{2 / 3}}
$$

where $m_{\mathrm{E}}, v_{\mathrm{E}}$, and $(\mathrm{d} v / \mathrm{d} t)_{\mathrm{E}}$ are the mass, velocity and deceleration at the terminal point, respectively. $\rho_{\mathrm{m}}$ is the meteoroid density and $\rho_{\mathrm{E}}$ the air density at the terminal height. The latter was again calculated by using the US standard atmosphere model (US Standard Atmosphere 1976).

For the "Sevilla" fireball the calculation of the atmospheric trajectory yields $v_{\mathrm{E}}=8.3 \mathrm{~km} \mathrm{~s}^{-1}$ and $(\mathrm{d} v / \mathrm{d} t)_{\mathrm{E}}=-23.8 \mathrm{~km} \mathrm{~s}^{-2}$, so the calculated terminal mass is $m_{\mathrm{E}}=14 \pm 6 \mathrm{~g}$ for a meteor density $\rho_{\mathrm{m}}=2.4 \mathrm{~g} \mathrm{~cm}^{-3}$ and $6 \pm 3 \mathrm{~g}$ for $\rho_{\mathrm{m}}=3.7 \mathrm{~g} \mathrm{~cm}^{-3}$. For the "Fuencaliente" event the analysis of the trajectory provided 
Table 5. Derived elemental abundances relative to Si of the two fireballs discussed here compared with the inferred for other solar system undifferentiated materials.

\begin{tabular}{lccccccc}
\hline \hline & $\mathrm{Mg}$ & $\mathrm{Na}$ & $\mathrm{Ca}$ & $\mathrm{Ni} \times 10^{-3}$ & $\mathrm{Mn} \times 10^{-4}$ & $N\left(\mathrm{~cm}^{-2}\right)$ & $T(\mathrm{~K})$ \\
\hline SPMN210712 "Fuencaliente" & 0.90 & 0.025 & 0.045 & 42 & 78 & $10^{15}$ & 4800 \\
SPMN291212 "Sevilla" & 1.05 & 0.055 & 0.014 & 30 & 92 & $5 \times 10^{14}$ & 4600 \\
1P/Halley & 0.54 & 0.054 & 0.034 & 22 & 30 & - & - \\
IDPs & 0.85 & 0.085 & 0.048 & 37 & 150 & - & - \\
CI chondrites & 1.06 & 0.060 & 0.071 & 51 & 90 & - & - \\
CM chondrites & 1.04 & 0.035 & 0.072 & 46 & 60 & - & - \\
\hline
\end{tabular}

References. Jessberger et al. (1988); Rietmeijer \& Nuth (2000); Rietmeijer (2002); Trigo-Rodríguez (2002); Trigo-Rodríguez et al. (2003).

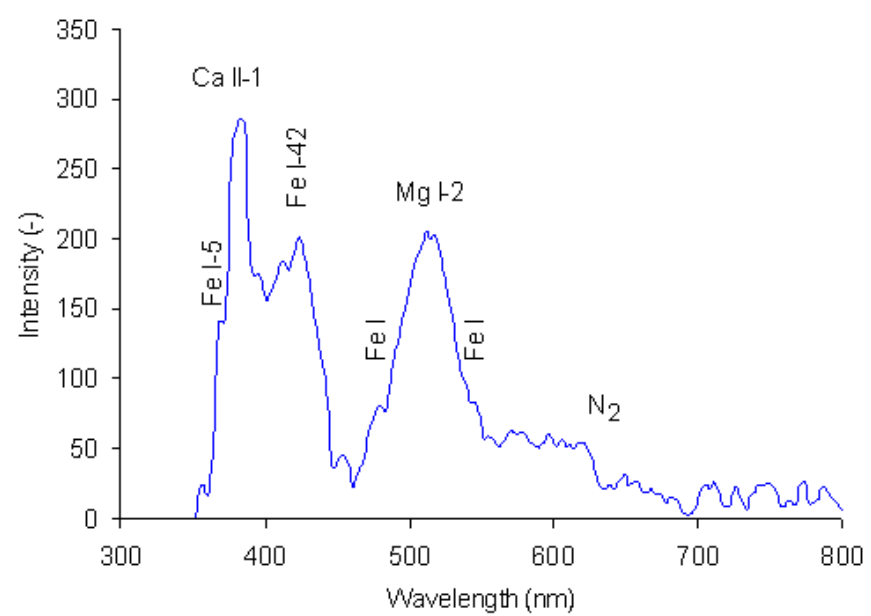

Fig. 5. Calibrated emission spectrum recorded for the SPMN 210712 "Fuencaliente" bolide. Intensity is expressed in arbitrary units.

the values $v_{\mathrm{E}}=8.1 \mathrm{~km} \mathrm{~s}^{-1}$ and $(\mathrm{d} v / \mathrm{d} t)_{\mathrm{E}}=-9.9 \mathrm{~km} \mathrm{~s}^{-2}$, and the terminal mass of the meteoroid yields $m_{\mathrm{E}}=78 \pm 23 \mathrm{~g}$ for $\rho_{\mathrm{m}}=2.4 \mathrm{~g} \mathrm{~cm}^{-3}$ and $33 \pm 11 \mathrm{~g}$ for $\rho_{\mathrm{m}}=3.7 \mathrm{~g} \mathrm{~cm}^{-3}$. Since these terminal masses are very low, no meteorite search was organized.

\subsection{Emission spectra and inferred chemical abundances}

Our video spectrographs recorded the emission spectrum for both fireballs. These were used to obtain insight into the chemical nature of the parent meteoroids. We employed our CHIMET software, which follows the technique described in Borovicka (1993) and Trigo-Rodriguez (2002), to reduce these spectra (Madiedo et al. 2011b, 2013a,b). Thus, we first deinterlaced the video sequences containing the emission spectrum. The frames in the resulting video files were then dark-frame substracted and flat-fielded. Next, the signal was calibrated in wavelengths by identifying typical lines appearing in meteor spectra and corrected by taking the efficiency of the recording instrument into account. The result can be seen in Figs. 5 and 6.

As can be seen in Fig. 5, the most prominent emission lines of the SPMN210712 "Fuencaliente" bolide spectrum correspond to multiplets Ca II-1 (at 393-397 nm), Fe I-42 (438 nm), and Mg I-2 (518 nm). Other Fe I lines are visible all along the spectrum. The Na I-1 line at $589 \mathrm{~nm}$ is almost missing, barely visible over the background. From this spectrum we suspect that the meteoroid was depleted in $\mathrm{Na}$ for some reason. Sometimes it has been found that fragile meteoroids suffer preferential ablation of $\mathrm{Na}$ in the highest parts of their luminous trajectories (Madiedo et al. 2013c). Another possibility could be heating experienced

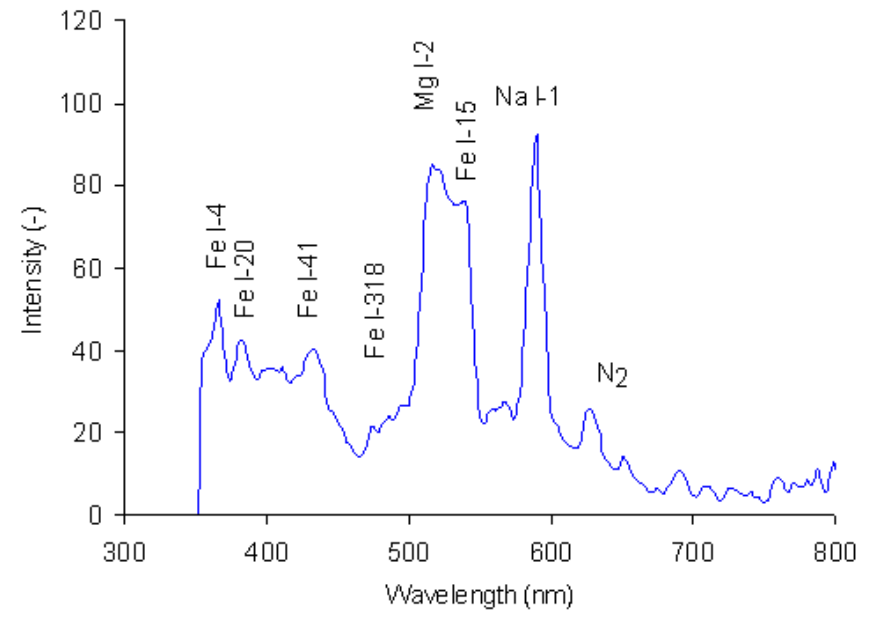

Fig. 6. Calibrated emission spectrum recorded for the SPMN291212 "Sevilla" fireball. Intensity is expressed in arbitrary units.

during close approaches to the Sun, but this seems unlikely due to the inferred meteoroid mass and relative high value of the perihelion distance $(q=0.929 \pm 0.002)$. The rest of the spectrum looks quite normal for a meteoroid of chondritic nature but low geocentric velocity, as is also inferred from the comparison between the computed chemical abundance ratios relative to $\mathrm{Si}$ summarized in Table 5. On the other hand, the emission spectrum of the SPMN291212 "Sevilla" fireball is characteristic of a low geocentric velocity event (Trigo-Rodríguez 2002; Trigo-Rodríguez et al. 2003). In these particular cases, the abundance of elements forming part of refractory phases, like $\mathrm{Ca}$ and $\mathrm{Al}$, are underestimated. This is because it is inefficient to bring all available $\mathrm{Ca}$ or $\mathrm{Al}$ to the vapor phase. Thus, we previously noted (Trigo-Rodríguez et al. 2003) that in chondritic meteoroids the elements $\mathrm{Ca}$ and $\mathrm{Al}$ are associated with refractory minerals that are not fully vaporized during atmospheric ablation (Trigo-Rodríguez 2002; Trigo-Rodríguez et al. 2003, 2004; Trigo-Rodríguez \& Llorca 2007). Consequently, it is not surprising that the contribution of Ca II-1 (at 393-397 nm) is almost indistinguishable, since it is blended with the Fe I-4, Fe I-20 and Fe I-45 multiplets. In any case, the spectrum is dominated by the Fe I-42 (438 nm), Mg I-2 (518 nm), Fe I-15 (537 nm), and Na I-1 $(589 \mathrm{~nm})$ multiplets. In the red region of the spectrum we have identified atmospheric $\mathrm{N}_{2}$ bands around $626 \mathrm{~nm}$. In general, the chemical ratios relative to $\mathrm{Si}$ for this meteoroid fall within the values expected for chondritic meteorites (Table 5). 


\section{Conclusions}

We have analyzed two fireballs of sporadic origin observed over Spain in 2012. These were catalogued as SPMN210712 "Fuencaliente" and SPMN291212 "Sevilla". Our main conclusions are as follows.

1. Our analysis shows that the body that produced the "Fuencaliente" fireball had a preatmospheric mass of $212 \pm$ $20 \mathrm{~kg}$. This mass was $4.1 \pm 0.5 \mathrm{~kg}$ for the "Sevilla" event. The inferred orbital parameters reveal a likely asteroidal origin for the "Fuencaliente" meteoroid. On the other hand, the "Sevilla" meteoroid followed a JFC orbit before impacting the atmosphere. Their tensile strength was inferred from the aerodynamic pressure at which these particles fragmented in the atmosphere. The derived toughness of the "Sevilla" meteoroid suggests that this particle was produced by an evolved comet or that the meteoroid was ejected from that body long ago.

2. Both bolides exhibited fast and quasi-periodic variations in brightness along their atmospheric path. These, however, were produced by different mechanisms. Thus, for the "Sevilla" fireball the observed flickering behavior was caused by the rotation of the non-spherical parent meteoroid. For the "Fuencaliente" event, a successive detachment of fragments would be responsible for the observed quasiperiodic variations of brightness.

3. The calculation of the atmospheric trajectory reveals that these deep-penetrating fireballs ended their luminous phase at a height of $29.0 \pm 0.5$ and $30.7 \pm 0.5 \mathrm{~km}$ above the ground level. A non-zero terminal mass was inferred for both events, which shows that these bolides were meteorite-droppers. However, since the inferred mass is low (below 100 grams), no meteorite search was organized.

4. Both fireballs probably were chondritic in composition, but exhibiting some peculiarities. For example, the emission spectrum of the "Fuencaliente" fireball reveals Na depletion like the one predicted for a meteoroid suffering preferential ablation in the highest parts of the trajectory. Another possibility could be the heating experienced during close approaches to the Sun, but this seems unlikely due to the inferred meteoroid mass and relatively far perihelion distance. On the other hand, the "Sevilla" event has weaker emission lines probably due to its very low geocentric velocity that also participates in obtaining low chemical ratios for elements forming refractory minerals.

Acknowledgements. We acknowledge support from the Spanish Ministry of Science and Innovation (project AYA2011-26522) and Junta de Andalucía (project P09-FQM-4555). We also thank the AstroHita Foundation for its support in the establishment and operation of the automated meteor observing station located at La Hita Astronomical Observatory (La Puebla de Almoradiel, Toledo, Spain).

\section{References}

Adolfsson, L. G., \& Gustafson, B. A. S. 1994, P\&SS, 42, 593

Babadzhanov, P. B., \& Konovalova, N. A. 2004, A\&A, 428, 241

Beech, M. 2001, MNRAS, 326, 937

Beech, M., \& Brown, P. 2000, Planet. Space Sci., 48, 925

Beech, M., Illingworth, A., \& Murray, S. 2003, Meteor. Planet. Sci., 38, 1045

Borovička, J. 1993, A\&A, 279, 627

Bronshten, V. A. 1981, Geophys. Astrophys. Monographs (Dordrecht: Reidel)

Brown, P. et al. 1998, Nature, 37, 624

Campbell, M. D., Brown, P. G., Leblanc, A. G., et al. 2000, Meteor. Planet. Sci., 35,1259

Čapek, D., \& Vokrouhlický, D. 2004, Icarus, 172, 526

Ceplecha, Z. 1987, Bull. Astron. Inst. Cz., 38, 222

Ceplecha, Z., \& McCrosky, R. E. 1976, J. Geophys. Res., 81, 6257

Ceplecha, Z., Novakova-Jezkova, M., Porubcan, V., et al. 1983, Bull. Astron. Inst. Cz., 34, 195

Getman, V. S. 1993, in Meteoroids and their Parent Bodies. Astronomical Inst. Slovak. Acad. Sci., Bratislava, eds. I. Stohl, \& I. P. Williams, 307

Halliday, I. 1963, Smithson. Contrib. Astrophys., 7, 161

Hawkes, R. L., \& Jones, J. 1975, MNRAS, 173, 339

Hughes, D. W. 1978, in Cosmic Dust, ed. J. A. M. McDonnell (Chichester: Wiley and Sons), 167

Jenniskens P. 2006, Meteor Showers and their Parent Comets (Cambridge University Press)

Jessberger, E. K., Christoforidis, A., \& Kissel, J. 1988, Nature, 332, 691

Konovalova, N. A. 2003, A\&A, 404, 1145

Kruchinenko, V. G. 1995, Sol. Syst. Res., 29, 309

Lebedinets, V. N. 1986, Dokl. AN SSSR, 291, 313

Madiedo, J. M., \& Trigo-Rodríguez, J. M. 2008, Earth Moon Planets, 102, 133

Madiedo, J. M., Trigo-Rodríguez, J. M., Ortiz, J. L., \& Morales, N. 2010, Adv. Astron., 2010, 1

Madiedo, J. M., Trigo-Rodríguez, J. M., \& Lyytinen, E. 2011a, NASA/CP-2011216469, 330

Madiedo, J. M., Toscano, F. M., \& Trigo-Rodríguez, J. M. 2011b, EPSC-DPS Joint Meeting 2011, Abstract \#Vol. 6, EPSC-DPS2011-72

Madiedo, J. M., Trigo-Rodríguez J. M., Lyyttinen, E., et al. 2013a, MNRAS, DOI: $10.1093 / \mathrm{mnras} / \mathrm{stt} 288$

Madiedo, J. M., Trigo-Rodríguez, J. M., Williams, I. P., Ortiz, J. L., \& Cabrera, J. 2013b, MNRAS, DOI: 10.1093/mnras/stt342

Madiedo J. M. et al. 2013c, MNRAS, submitted

Murray, I. S., Hawkes, R. L., \& Jenniskens, P. 1999, Meteorit., \& Planet. Sci., 34, 949

Novikov, G. G., Pecina, P., \& Konovalova, N. A. 1998, A\&A, 329, 769

Oleak, N. 1964, Astron. Nachr., 228, 7

Olsson-Steel, D. I. 1987, MNRAS, 226, 1

Opik, E. 1936, Publ. Tartu Obs., 28, 13

Rietmeijer, F. 2002, Chemie der Erde, 62, 1

Rietmeijer, F., \& Nuth, J. A. 2000, Earth, Moon Planets, 82, 325

Trigo-Rodríguez, J. M. 2002, Ph.D. Thesis, Universitat de Valencia

Trigo-Rodríguez, J. M., \& Llorca, J. 2006, MNRAS, 372, 655

Trigo-Rodríguez, J. M., \& Llorca, J. 2007, MNRAS, 375, 415

Trigo-Rodríguez, J. M., Llorca, J., Borovička, J., \& Fabregat, J. 2003, Meteorit. Planet. Sci., 38, 1283

Trigo-Rodríguez, J. M., Llorca, J., Borovicka, J., \& Fabregat, J. 2004, Earth Moon Planets, 95, 375

Trigo-Rodríguez, J. M., Madiedo, J. M., Llorca, J., et al. 2007, MNRAS, 380, 126

Trigo-Rodríguez, J. M., Madiedo, J. M., Williams, I. P., \& Castro-Tirado, A. J. 2009, MNRAS, 392, 367

US Standard Atmosphere 1976, NOA-NASA-USAF, Washington 